\title{
A DNS approach to stability study about a supersonic mixing layer flow
}

\author{
F. Guan, Q. Wang, N. Zhu, Z. Li \& Q. Shen \\ CAAA (China Academy of Aerospace Aerodynamic), Beijing, China
}

\begin{abstract}
In this paper linear and nonlinear issues of a mixing layer at $M c=1.2$ are studied with a DNS method. Navier-Stokes equations in perturbation form are solved with a finite difference method of third-order accuracy. An approximated boundary condition treatment of small disturbance along the outside boundary is proposed on flow characteristics. This boundary condition is verified to be valid in the numerical case. Linear issue of a mixing layer at $M c=1.2$ is simulated. Three modes of instability in the mixing layer have been simulated: Slow-Mode, Fast-Mode and Mix-Mode. Nonlinear issues of the mixing layer at $M c=1.2$ are also studied. The mode transition of the mixing layer instability is simulated. At low-frequency disturbance, simple mode instability develops in the mixing layer upstream, which is Slow-Mode instability. In the middle of the mixing layer, the Mix-Mode instability develops, and Mix-Mode instability are shifting to FastMode in the downstream.
\end{abstract}

Keywords: mixing layer, DNS, boundary condition, acoustic radiation vortex mod, mode transition.

\section{Introduction}

Instability of a supersonic mixing layer is a fundamental phenomenon leading to turbulent flow. It is important to study the production and evolution of two-dimensional and three-dimensional unstable structures in a planar supersonic mixing layer to understand the mechanism of transition. It is known that there are several ways leading to the development of three-dimensional structures. Secondary instability is a natural way to make three-dimensional structures and then to transition. 
DNS (direct numerical simulation) is a useful tool to study the instability of the mixing layer. Boundary condition's treatment is an important technique for DNS to resolve the compressible N-S equations in the study of supersonic mixing layer instability and transition. Inadequate boundary condition's treatment will introduce the non-physics fluctuations from the boundary of the flow field, and affect the natural instability of supersonic shear layer. The present work is focused on the secondary instability and the boundary condition's treatment in DNS of the supersonic mixing layer.

In decades, many studies about shear layer instability have been made. It is well known that the supersonic shear layer instabilities are Kelvin-Helmholtz instabilities from the incompressible flow that is involved with vortex roll-ups and pairing, and which is also a kind of Tollmien-Schlichting wave.

In 1984, W.S. Saric, V.V. Kozlov and V.Y. Levchenko studied the development of three-dimensional disturbance waves in incompressible boundary layers by visualization. They found that the two-dimensional T-S wave grows into three-dimensional structures in evolvement processes and the new three-dimensional perturbation wave is a periodic " $\lambda$ " vortex in streamwise and lateral direction. The three-dimensional instability waves are cataloged into three types: C-type [1], H-type [2] and K-type [3].

D. Papamochou and A. Roshoko [4] defined the convective Mach number, which is the parameter of the compressibility in the supersonic shear layer and proved that by experiment in 1986. The Mc has been found to be a key parameter to describe the compressibility of the flow.

In 1989, S. K. Lele [5] studied the compressible free shear flow with the DNS method. In his simulations on supersonic mixing layer flows of $M c<1.0$, vortex roll-up and pairing dominate the instability process. He also found shock-lets may emerge with vortex structure for $M c>0.7$.

In the year 2000, Q. Shen and H. Zhang [6] studied a spatially developed two-dimensional supersonic mixing layer at $M c=0.5$ with the DNS method, and found the intermittency takes place during the vortex unstable evolvement. The intermittent structure comes from nonlinear vortex roll-up and paring process that was analyzed by J. R. Luo et al [7]. The Lyapunov index and fractal dimension were calculated, and show a typical nonlinear growth.

In 2006, the secondary instability of a planar supersonic shear layer at $M c=0.5$ is simulated. The secondary instability is a nature transition phenomenon by Q. Shen et al [8]. In 2006, F.M. Guan and Q. Shen [9] made a DNS study on secondary instability in a planar supersonic shear lay at $M c=0.5$ and found the secondary instability take place along with the T-S wave evolution and the twodimensional vortex pairing are intermittent in the supersonic shear layer. In 2007, F.M. Guan et al [10] studied the K-type and H-type instability in a supersonic mixing layer, and found the secondary instability could be obviously hampered by vortex pairing.

In 2007, Q. Shen et al [11] had studied the mixing layer at Mc=1.2 on LST. They found that there are three modes of the mixing layer instability, which are Slow-Mode instability led by low frequency disturbance, Fast-Mode instability 
led by high frequency disturbance and Mix-Mode led by middle frequency disturbance.

In the present work, a boundary condition's treatment is studied to approach the supersonic shear layer instability in resolving the perturbation form N-S equations. Instability of a planar two-dimensional supersonic shear layer at $M c=1.2$ has been studied with this boundary condition's treatment, and the acoustic radiation vortex mode has been found. All of the numerical results of the supersonic shear layer instability indicate that the boundary conditions treatment is adequate.

\section{Governing equations and numerical method}

The compressible two-dimensional unsteady Navier-Stokes equations are:

where

$$
\frac{\partial \hat{Q}}{\partial t}+\frac{\partial \hat{E}}{\partial \xi}+\frac{\partial \hat{F}}{\partial \eta}=\frac{\partial \hat{E}_{v}}{\partial \xi}+\frac{\partial \hat{F}_{v}}{\partial \eta}
$$

$$
\begin{gathered}
\hat{Q}=\frac{1}{J}\left(\begin{array}{c}
\rho \\
\rho u \\
\rho v \\
e
\end{array}\right), \hat{E}=\frac{1}{J}\left(\begin{array}{c}
\rho U \\
\rho U u+\xi_{x} p \\
\rho U v+\xi_{y} p \\
(e+p) U
\end{array}\right), \hat{F}=\frac{1}{J}\left(\begin{array}{c}
\rho V \\
\rho V u+\eta_{x} p \\
\rho V v+\eta_{y} p \\
(e+p) V
\end{array}\right), \\
\hat{E}_{v}=\frac{1}{J \operatorname{Re}}\left(\begin{array}{c}
\xi_{x} \tau_{x x}+\xi_{y} \tau_{x y} \\
\xi_{x} \tau_{x y}+\xi_{y} \tau_{y y} \\
\xi_{x}\left(u \tau_{x x}+v \tau_{x y}-q_{x}\right)+\xi_{y}\left(u \tau_{x y}+v \tau y y-q_{y}\right)
\end{array}\right), \\
0 \\
\hat{F}_{v}=\frac{1}{J \operatorname{Re}}\left(\begin{array}{c}
\eta_{x} \tau_{x x}+\eta_{y} \tau_{x y} \\
\eta_{x} \tau_{x y}+\eta_{y} \tau_{y y} \\
\eta_{x}\left(u \tau_{x x}+v \tau_{x y}-q_{x}\right)+\eta_{y}\left(u \tau_{x y}+v \tau_{y y}-q_{y}\right)
\end{array}\right), \\
\tau_{x x}=\frac{2}{3} \mu\left(2 \frac{\partial u}{\partial x}-\frac{\partial v}{\partial y}\right), \tau_{x y}=\mu\left(\frac{\partial u}{\partial y}-\frac{\partial v}{\partial x}\right), \tau_{y y}=\frac{2}{3} \mu\left(2 \frac{\partial u}{\partial y}-\frac{\partial v}{\partial x}\right) \\
\frac{1}{2}\left(u^{2}+v^{2}\right)+\frac{1}{\gamma-1} p, \operatorname{Re}=\frac{\rho_{\infty} u_{\infty} L}{\mu_{\infty}}, \\
U=\xi_{x} u+\xi_{y} v, V=\eta_{x} u+\eta_{y} v
\end{gathered}
$$




$$
\begin{gathered}
q_{x}=-\frac{\mu}{(\gamma-1) M_{\infty}^{2} \operatorname{Pr}} \frac{\partial T}{\partial x}, q_{y}=-\frac{\mu}{(\gamma-1) M_{\infty}^{2} \operatorname{Pr}} \frac{\partial T}{\partial y} \\
T=\gamma M_{\infty}^{2} \frac{p}{\rho}, \mu=T^{\frac{3}{2}} \frac{1+C}{C+T} \text { and } C=\frac{110.4 K}{T_{\infty}} .
\end{gathered}
$$

In the study of supersonic shear layer instability, the flow field is computed in two processes that first one is the study undisturbed flow field computation and the second one is unsteady disturbed flow field computation. Assuming undisturbed flow is steady state, as $\hat{E}=\hat{E}_{0}, \hat{F}=\hat{F}_{0}, \hat{E}_{v}=\hat{E}_{v 0}, \hat{F}_{v}=\hat{F}_{v o}$, which satisfy steady Navier-Stokes equations as

$$
\frac{\partial \hat{E}_{0}}{\partial \xi}+\frac{\partial \hat{F}_{0}}{\partial \eta}=\frac{\partial \hat{E}_{v 0}}{\partial \xi}+\frac{\partial \hat{F}_{v 0}}{\partial \eta}
$$

where subscript " 0 " is denoted for steady state i.e. base flow, the flow can be taken as the combination of the base flow and the perturbation flow. Denoting the perturbation variables with prime, then it can be taken that $\hat{Q}=\hat{Q}_{0}+\hat{Q}^{\prime}$,

$$
\begin{gathered}
\hat{E}=\hat{E}_{0}+\hat{E}^{\prime}, \hat{F}=\hat{F}_{0}+\hat{F}^{\prime}, \hat{E}_{V}=\hat{E}_{v 0}+\hat{E}_{v}^{\prime}, \hat{F}_{v}=\hat{F}_{v 0}+\hat{F}_{v}^{\prime} \text {, and } \\
\frac{\partial \hat{Q}^{\prime}}{\partial t}+\frac{\partial \hat{E}^{\prime}}{\partial \xi}+\frac{\partial \hat{F}^{\prime}}{\partial \eta}=\frac{\partial \hat{E}_{v}^{\prime}}{\partial \xi}+\frac{\partial \hat{F}^{\prime}}{\partial \eta}
\end{gathered}
$$

where

$$
\begin{aligned}
& \hat{Q}^{\prime}=\frac{1}{J}\left(\begin{array}{c}
\rho^{\prime} \\
(\rho u)^{\prime} \\
(\rho v)^{\prime} \\
e^{\prime}
\end{array}\right), \hat{E}^{\prime}=\frac{1}{J}\left(\begin{array}{c}
\rho_{0} U^{\prime}+\rho^{\prime}\left(U_{0}+U^{\prime}\right) \\
\rho_{0} u_{0} U^{\prime}+(\rho u)^{\prime}\left(U_{0}+U^{\prime}\right)+\xi_{x} p^{\prime} \\
\rho_{0} v_{0} U^{\prime}+(\rho v)^{\prime}\left(U_{0}+U^{\prime}\right)+\xi_{y} p^{\prime} \\
\left(e^{\prime}+p^{\prime}\right)\left(U_{0}+U^{\prime}\right)+\left(e_{0}+p_{0}\right) U^{\prime}
\end{array}\right), \\
& \hat{F}^{\prime}=\frac{1}{J}\left(\begin{array}{c}
\rho_{0} V^{\prime}+\rho^{\prime}\left(V_{0}+V^{\prime}\right) \\
\rho_{0} u_{0} V^{\prime}+(\rho u)^{\prime}\left(V_{0}+V^{\prime}\right)+\eta_{x} p^{\prime} \\
\rho_{0} v_{0} V^{\prime}+(\rho v)^{\prime}\left(V_{0}+V^{\prime}\right)+\eta_{y} p^{\prime} \\
\left(e^{\prime}+p^{\prime}\right)\left(V_{0}+V^{\prime}\right)+\left(e_{0}+p_{0}\right) V^{\prime}
\end{array}\right), \\
& \hat{E}_{v}^{\prime}=\frac{1}{J \operatorname{Re}}\left(\begin{array}{c}
0 \\
\xi_{x} \tau_{x x}^{\prime}+\xi_{y} \tau_{x y}^{\prime} \\
\xi_{x} \tau_{x y}^{\prime}+\xi_{y} \tau_{y y}^{\prime} \\
\xi_{x}\left[\left(u \tau_{x x}\right)^{\prime}+\left(v \tau_{x y}\right)^{\prime}-q_{x}^{\prime}\right]+\xi_{y}\left[\left(u \tau_{x y}\right)^{\prime}+\left(v \tau_{y y}\right)^{\prime}-q_{y}^{\prime}\right]
\end{array}\right),
\end{aligned}
$$




$$
\begin{aligned}
& \hat{F}_{v}^{\prime}=\frac{1}{J \operatorname{Re}}\left(\begin{array}{c}
0 \\
\eta_{x} \tau_{x x}^{\prime}+\eta_{y} \tau_{x y}^{\prime} \\
\eta_{x} \tau_{x y}^{\prime}+\eta_{y} \tau_{y y}^{\prime} \\
\eta_{x}\left[\left(u \tau_{x x}\right)^{\prime}+\left(v \tau_{x y}\right)^{\prime}-q_{x}^{\prime}\right]+\eta_{y}\left[\left(u \tau_{x y}\right)^{\prime}+\left(v \tau_{y y}\right)^{\prime}-q_{y}^{\prime}\right]
\end{array}\right), \\
& e^{\prime}=\frac{1}{2} \rho_{0}\left[\left(2 u_{0}+u^{\prime}\right) u^{\prime}+\left(2 v_{0}+v^{\prime}\right) v^{\prime}\right]+\frac{1}{2} \rho^{\prime}\left[\left(u_{0}+u^{\prime}\right)^{2}+\left(v_{0}+v^{\prime}\right)^{2}\right]+\frac{p^{\prime}}{\gamma-1} \\
& \tau_{x x}^{\prime}=\frac{2}{3}\left[\left(\mu_{0}+\mu^{\prime}\right)\left(2 \frac{\partial u^{\prime}}{\partial x}-\frac{\partial v^{\prime}}{\partial y}\right)+\mu^{\prime}\left(2 \frac{\partial u_{0}}{\partial x}-\frac{\partial v_{0}}{\partial y}\right)\right], \\
& \tau_{x y}^{\prime}=\left(\mu_{0}+\mu^{\prime}\right)\left(\frac{\partial u^{\prime}}{\partial y}-\frac{\partial v^{\prime}}{\partial x}\right)+\mu^{\prime}\left(\frac{\partial u_{0}}{\partial y}-\frac{\partial v_{0}}{\partial x}\right) \text {, } \\
& \tau_{y y}^{\prime}=\frac{2}{3}\left[\left(\mu_{0}+\mu^{\prime}\right)\left(2 \frac{\partial v^{\prime}}{\partial y}-\frac{\partial u^{\prime}}{\partial x}\right)+\mu^{\prime}\left(2 \frac{\partial v_{0}}{\partial y}-\frac{\partial u_{0}}{\partial x}\right)\right], \\
& q_{x}^{\prime}=-\frac{1}{(\gamma-1) M_{\infty}^{2} \operatorname{Pr}}\left[\left(\mu_{0}+\mu^{\prime}\right) \frac{\partial T^{\prime}}{\partial x}+\mu^{\prime} \frac{\partial T^{\prime}}{\partial x}\right] \text {, } \\
& q_{y}^{\prime}=-\frac{1}{(\gamma-1) M_{\infty}^{2} \operatorname{Pr}}\left[\left(\mu_{0}+\mu^{\prime}\right) \frac{\partial T^{\prime}}{\partial y}+\mu^{\prime} \frac{\partial T^{\prime}}{\partial y}\right] \text {, } \\
& T^{\prime}=T_{0}\left(\frac{p^{\prime}}{p_{0}}-\frac{\rho^{\prime}}{\rho_{0}}\right), \mu^{\prime}=\mu_{0}\left(\frac{3}{2 T_{0}}-\frac{1}{C+T_{0}}\right) T^{\prime}, \\
& \left(u \tau_{x x}\right)^{\prime}=\left(\mu_{0}+\mu^{\prime}\right)\left[\left(u_{0}+u^{\prime}\right) \tau_{x x}^{\prime}+u^{\prime} \tau_{x x 0}\right]+\mu^{\prime} u_{0} \tau_{x x 0}, \\
& \left(u \tau_{x y}\right)^{\prime}=\left(\mu_{0}+\mu^{\prime}\right)\left[\left(u_{0}+u^{\prime}\right) \tau_{x y}^{\prime}+u^{\prime} \tau_{x y 0}\right]+\mu^{\prime} u_{0} \tau_{x y 0}, \\
& \left(v \tau_{x y}\right)^{\prime}=\left(\mu_{0}+\mu^{\prime}\right)\left[\left(v_{0}+v^{\prime}\right) \tau_{x y}^{\prime}+v^{\prime} \tau_{x y 0}\right]+\mu^{\prime} v_{0} \tau_{x y 0}, \\
& \left(v \tau_{y y}\right)^{\prime}=\left(\mu_{0}+\mu^{\prime}\right)\left[\left(v_{0}+v^{\prime}\right) \tau_{y y}^{\prime}+v^{\prime} \tau_{y y 0}\right]+\mu^{\prime} v_{0} \tau_{y y 0} \text {, }
\end{aligned}
$$

Once $Q^{\prime}$ is solved from eqn (3), the flow variables can be deciphered as followings $u^{\prime}=\frac{(\rho u)^{\prime}-\rho^{\prime} u_{0}}{\rho_{0}+\rho^{\prime}}, v^{\prime}=\frac{(\rho v)^{\prime}-\rho^{\prime} v_{0}}{\rho_{0}+\rho^{\prime}}$. Eqn (3) are the governing equations, which define the perturbation waves travelling within the base flow.

These equations are solved using a finite difference method [8] which is the third-order accuracy in spatial and in temporal direction. 


\section{Boundary conditions}

The boundary conditions treatment in compressible Navier-Stokes equations had been mature. The boundary conditions treatment is according to the direction of disturbance propagation in the compressible Navier-Stokes equations. In twodimensional unsteady compressible Navier-Stokes equations, there are four eigenvalues of $\frac{\partial \hat{E}}{\partial \hat{Q}}$ in $\xi$ direction which are $\lambda_{1}=\lambda_{2}=U, \lambda_{3}=U+c$, $\lambda_{4}=U-c$, and four eigenvalues of $\frac{\partial \hat{F}}{\partial \hat{Q}}$ in $\eta$ direction which are $\lambda_{1}=\lambda_{2}=V, \lambda_{3}=V+c, \lambda_{4}=V-c . c$ is the acoustic speed, $c=\sqrt{\gamma \frac{p}{\rho}}$.

In the supersonic flow field, the four eigenvalues on stream wise are all positive and one eigenvalue on flow normal direction is positive. There three eigenvalues on stream wise are positive and one eigenvalue on flow normal direction is positive in subsonic flow.

The schematic of a mixing layer is shown in fig. 1. On the inflow boundary, the flow is supersonic and flow variables are constants and equal to incoming flow parameters. In supersonic, extrapolation is usually used to get the flow variables on the outflow boundary. The top and bottom boundaries are free flow conditions. For the undisturbed base flow, $\lambda_{1}=\lambda_{2}=V=0$, $\lambda_{3}=V+c>0$ and $\lambda_{4}=V-c<0$.

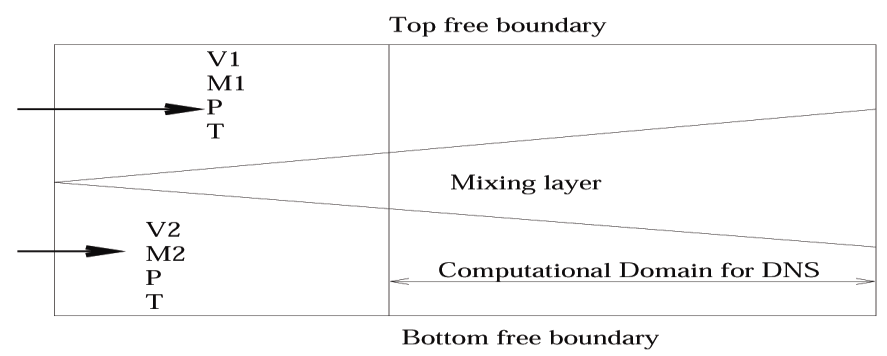

Figure 1: Schematic of a mixing layer.

For the perturbation flow, the disturbance assumes to travel from free boundary to the outside. Outside the mixing layer, the amplitude of the disturbance wave is quite small. These small disturbance waves are assumed as adiabatic. With this assumption $u^{\prime}, v^{\prime}$ and $p^{\prime}$ are obtained by extrapolations. $\rho^{\prime}$ is obtained from adiabatic relation as:

$$
d \rho=\frac{1}{\gamma} \rho \frac{d p}{p}
$$


By this approximation, the non-reflecting boundary condition is obtained. This relation is to be verified in the next computational case.

\section{Acoustic radiation vortex mode in two-dimensional supersonic shear layer at $M c=1.2$}

A computational case is set which is a supersonic free mixing layer at $M c=1.2$. In present computational cases, $M_{l}=3.5, M_{2}=1.1$ are taken for two flow streams. Other flow conditions are $T_{\infty}=1200.0 .0 \mathrm{~K}$ for flow temperature, $\rho_{\infty}=1.0$ for non-dimensional flow density, and $R e_{L}=1.0 \mathrm{E}+6$ with reference length $\mathrm{L}$.

The undisturbed steady flow field is obtained by resolving the steady Navier-Stokes equation with a second order accurate NND scheme. The base flow used for DNS computation is cut from this flow field. The profile of base flow at $x / L=1.0$ which gives $u, \rho$ and $p$ by $y$ is shown in fig.2.

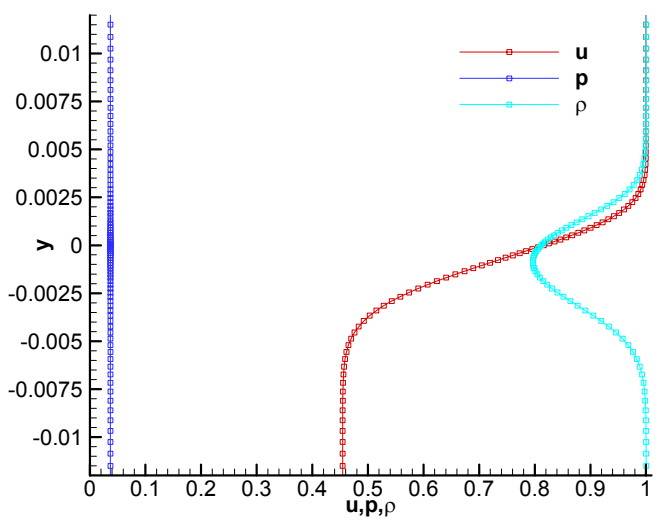

Figure 2: $\quad$ Steady mean flow profiles at $\mathrm{x}=1.0$.

\subsection{Linear simulation}

One case is set which is used to simulate linear development of a disturbance wave in the mixing layer. The computation region is $1.0<x<2.5$ in stream wise and $-0.188<y<0.188$ in lateral direction. In the present computation, $205 \times 201$ grids in $\mathrm{x}, \mathrm{y}$ directions are used, shown in fig. 1. A fundamental frequency disturbance wave in lateral velocity is introduced in the central point of the inflow boundary, which is:

$$
v^{\prime}=\varepsilon \sin (\varpi t)
$$

There, the amplitude of the disturbance wave is $\varepsilon=10^{-5}$, and the circular frequency of the disturbance wave is $\varpi$. Three circular frequencies have been chosen in present computational case, which are $\varpi=20,40,50$. The results are shown in fig. 3 . 


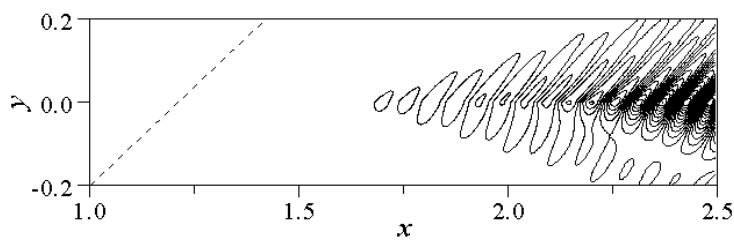

(a) $\varpi=20.0$

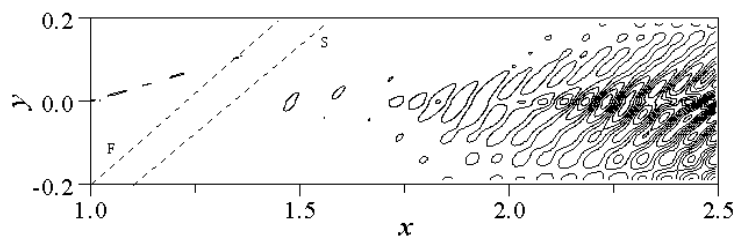

(b) $\varpi=40.0$

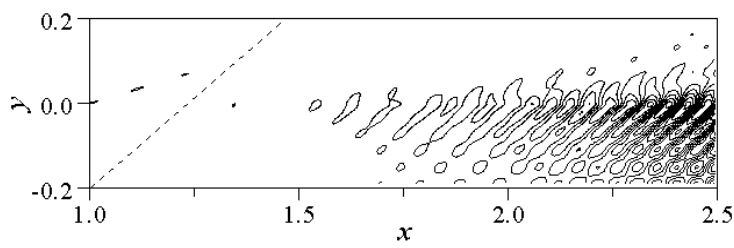

(c) $\varpi=50.0$

Figure 3: Contours of perturbation pressure $p^{\prime}$.

In the downstream of the mixing layer, three disturbance waves with different frequency all lead the expansion/compression wave's structures. This flow structure is a radiation characteristic, and will suppress the rate of mixing layer growth. Especially, three flow structures are obvious different radiation characteristic, that the flow structure led by low frequency disturbance wave radiates in the region of high velocity, the flow structure led by high frequency disturbance wave radiates in the region of low velocity and the flow structure led by middle frequency disturbance wave radiates in both high velocity region and low velocity region. It is indicated that the response of the supersonic mixing layer to disturbance wave depends on the frequency of disturbance wave. We name the flow structure led by low frequency disturbance Slow-Mode, the flow structure led by high frequency disturbance Fast-Mode and the flow structure led by middle frequency disturbance Mix-Mode.

\subsection{Nonlinear simulation}

The other case is set which is used to simulate the nonlinear development of the disturbance wave in the mixing layer. The computation region is $1.0<x<5.0$ in stream wise and $-0.25<y<0.25$ in lateral direction. In the present computation, $4001 \times 201$ grids in $\mathrm{x}, \mathrm{y}$ directions are used, shown in fig. 1. A fundamental 
frequency disturbance wave in lateral velocity is introduced in the central point of the inflow boundary, which is:

$$
v^{\prime}=\varepsilon \sin (\varpi t)
$$

There, the amplitude of the disturbance wave is $\varepsilon=10^{-3}$, and the circular frequency of the disturbance wave is $\varpi=30$. The results are shown in fig. 4 .

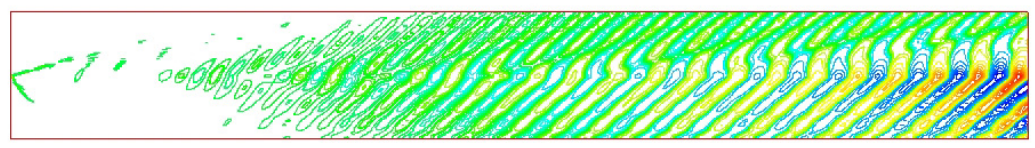

Figure 4: Disturbance pressure $p^{\prime}$ contours.

In the linear simulation, the low frequency of disturbance wave will lead the Slow-Mode. In the nonlinear computation, the thickness of the mixing layer grows in stream wise, and the response frequency to simple frequency rises in stream wise. So, the low frequency disturbance wave leads the Slow-Mode in inflow region of the mixing layer, the Mix-Mode in the middle region of the mixing layer. In the end of the mixing layer, the flow structure is that which the Fast-Mode dominates, shown in fig. 4.

Otherwise, the far field boundary conditions treatment in the present is verified in this computation, shown in fig. 5. Firstly, the phase of $p^{\prime}$ is the same as the phase of $\rho^{\prime}$. Secondly, flow variables of a point arbitrary selected from the mixing layer flow field, which is far from the centre of mixing layer and far from the far free boundary, is studied. In this case, a point studied at (3.145,$0.159)$ in the flow field is selected and the flow variables on it are:

$$
p^{\prime}=7.07 e-4, p=0.0258, \rho^{\prime}=8.58 e-4, \rho=1.0
$$

It can be found from these values that $\frac{p^{\prime}}{p} / \frac{\rho^{\prime}}{\rho}=1.4$. This result indicates that it is an adiabatic process and the boundary condition given by eqn. (4) is valid.

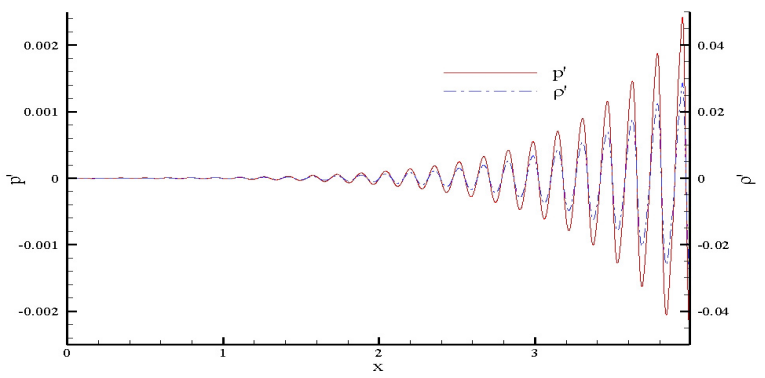

Figure 5: Perturbation pressure and density outside the mixing layer $(y=-0.159)$. 


\section{Conclusion}

An approximated boundary condition treatment of small disturbance outside the boundary is proposed based on flow characteristic.

Linear issues of a mixing layer at $M c=1.2$ are simulated on the DNS method. Three modes of instability in the mixing layer are simulated, which are Slow-Mode, Fast-Mode and Mix-Mode.

Nonlinear issues of the mixing layer at $M c=1.2$ are also studied. The instability mode transition of the mixing layer is found. At low frequency disturbance, simple instability mode develops in the mixing layer upstream, which is Slow-Mode instability. In the middle of the mixing layer, the MixMode instability develops, and Mix-Mode instability are shifting to Fast-Mode in the downstream.

\section{References}

[1] Craik A D D. "Non-liner resonant instability in bound layer," JFM, Vol. 40, 1971, pp. 393-413.

[2] Herbert Th. "Secondary instability of plane channel flow to subharmonic three-dimensional disturbances," Physic of Fluid, Vol. 63, 1983, pp. 871974.

[3] Kachanov $\mathrm{Yu} \mathrm{S}$, "On the resonant nature of the breakdown of laminar boundary layer,” JFM, Vol. 184. 1987, pp., 43-74.

[4] D. Papamoschou and A. Roshko, "Observation of supersonic free shear layers," AIAA Paper 86-0162, 1986.

[5] Lele, S. K., "Direct numerical simulation of compressible free shear flows," AIAA Paper 89-0374, 1989.

[6] Q. Shen, and H.X. Zhang, "Numerical simulation of intermittent structure in two-dimensional supersonic mixing-layer flow $(\mathrm{Mc}=0.5)$," Proceedings of the Fourth Asia Computational Fluid Dynamics Conference, edited by H. X. Zhang, UEST press, Chengdu (China), 2000, pp. 272-277.

[7] J. R. Luo, Q. Shen, and H. X. Zhang, "Analysis for nonlinear process of two-dimensional supersonic shear flow," Acta Aerodynamica Sinica, Vol. 20, No. 3, 2002 (in Chinese), pp. 282-288.

[8] Q. Shen, F.G. Zhuang, and F.M. Guan, "Numerical Simulation on a Planar Supersonic Free Shear Layer Secondary Instability", AIAA Paper 2006-3351, 2006.

[9] Guan F.M., Shen Q., "Direct numerical simulation on secondary instability in a planar supersonic shear lay at $\mathrm{Mc}=0.5$," The Eleventh Asian Congress of Fluid Mechanics, May, 2006. Malaysia.

[10] Guan F.M., Shen Q., Zhuang F.G., "Three-Dimension numerical simulation on controlled stability of a planar supersonic free shear layer", AIAA Paper 2007-1311.

[11] Q. Shen, Q. Wang, F.G. Zhuang. "Numerical analysis of acoustic radiation vortical modes in a spatial evolving supersonic plane shear layer", Chinese Journal of Theoretical and Applied Mechanics, Vol. 39, 2007, pp.7-14. 